\title{
Blow-analytic equivalence versus contact bi-Lipschitz equivalence
}

\author{
By Lev Birbrair, Alexandre Fernandes, Vincent Grandjean \\ and Terence Gaffney
}

(Received Feb. 2, 2016)

(Revised Dec. 6, 2016)

\begin{abstract}
The main result of this note is that two blow-analytically equivalent real analytic plane function germs are sub-analytically bi-Lipschitz contact equivalent.
\end{abstract}

\section{Introduction.}

The bi-Lipschitz classification of regular (analytic, smooth, definable) function germs is rather recent. Beyond the plane case, very little is known. As Parusiński and Henry showed in [10], the bi-Lipschitz right equivalence of real analytic plane function germs, unlike the classical case of the topological right equivalence [7], already presents moduli.

The blow-analytic equivalence (of real analytic function germs) introduced more than thirty years ago by Kuo [13], nevertheless has no moduli. Roughly speaking a blow-analytic homeomorphism is obtained by blowing-down an analytic isomorphism between blown-up manifolds (obtained by finite composition of blowings-up at the source and at the target respectively). Although not bi-Lipschitz in general it is still a rigid homeomorphism.

Birbrair, Fernandes and Grandjean, jointly with Gabrielov, recently exhibited a complete invariant, called minimal pizza, of the sub-analytic bi-Lipschitz contact equivalence of Lipschitz sub-analytic plane function germs [4]. The existence of this complete invariant implies that this equivalence has no moduli (result already known for analytic plane germs from a previous work of Birbrair and Fernandes, jointly with Costa and Ruas $[3]$ ). A pizza is a way to encode, by means of finitely many rational numbers, all the sub-analytic asymptotic behaviors of the considered function at the considered point. As the result of [4] states, such combinatorial data encoding the asymptotics of a given germ is indeed of metric nature. The local monomialization/resolution of (sub-)analytic function germs, although using a parameterization (the associated blowing-down mapping), is very convenient to investigate the sub-analytic asymptotic behaviors at the considered point. In the category of real analytic plane function germs, such hints suggest

2010 Mathematics Subject Classification. Primary 14P15, 14B05, 58K40; Secondary 32B20, 32S05, $32 \mathrm{~S} 15$.

Key Words and Phrases. blow-analytic, bi-Lipschitz, contact equivalence, blowing-up, pizza, width, Hsiang and Pati.

The first author was partially supported by CNPq-Brazil grant 300575/2010-6; The second author was partially supported by CNPq-Brazil grant 302998/2011-0; The fourth author was partially supported by PVE-CNPq Proc. 401565/2014-9. 
looking for relations between blow-analytic equivalence and bi-Lipschitz contact equivalence. Nevertheless Koike and Parusiński have already provided examples of bi-Lipschitz right-equivalent real analytic plane function germs (thus bi-Lipschitz contact equivalent) which are not blow-analytic equivalent $[\mathbf{1 5}]$.

This note establishes the relation between the blow-analytic equivalence and the sub-analytical bi-Lipschitz contact equivalence, namely

THEOREM 5.1. Blow-analytic equivalent real analytic plane function germs are subanalytically bi-Lipschitz contact equivalent.

The proof of this result is a consequence of the combinatorial local data of both blowings-up mapping in the definition of the (cascade) blow-analytic homeomorphism and of the combinatorial data of the blow-analytic equivalent function germs (once resolved). The analytic isomorphism inducing the homeomorphism preserves the combinatorial data of the pair "resolved function and corresponding resolution mapping" (one of the composition of blowings-up) and the other pair "resolved function and corresponding resolution mapping". The combinatorial data of a given pair function/resolution can be computed explicitly, using the parameterization in the resolved manifold, and this data can be used to produce all the ingredients that are needed to cook-up a pizza of the function. These ingredients encoded by finitely many rational numbers, are preserved by the blow-analytic homeomorphism, because of the isomorphism between the resolved manifolds preserving the combinatorial data of the pair function/resolution. More precisely our key argument is that a blow-analytic isomorphism preserves contact between any two real-analytic half-branches as well as the normalized order of a real analytic function along a real-analytic half-branch (see also [14]). When combined with an equivalent criterion of sub-analytic bi-Lipschitz contact equivalence, stated in Proposition 3.7, the blow-analytic homeomorphism maps a pizza (of one function) into an equivalent pizza (of the other function), so that Theorem 5.1 is true by the results of [4].

The paper is organized as follows.

Section 2 recalls quickly the notion of blow-analytic equivalence and two properties of metric nature. Section 3 presents the (sub-analytic) bi-Lipschitz contact equivalence with a new equivalence criterion, Proposition 3.7. It is followed by Section 4 where Lemma 4.1 shows the local normal form of any finite sequence of points blowings-up at any point of the exceptional divisor. This constitutes what we call the Hsiang and Pati (local) data of the blowing-down mapping, and further in this section we see how it reflects in any (cascade) blow-analytic homeomorphism. Section 5 provides a complete proof of the main result via several simple intermediary results leading to Proposition 5.2 stating that the considered blow-analytic homeomorphism induces an equivalence of Pizza, although stricto sensu not being one. The last section proposes a sketch of a proof of the existence of pizzas for plane real analytic function germs, providing clues why the result presented here was to be expected.

\section{Blow-analytic equivalence.}

We will only work with real analytic plane function germs, following the exposition of [14]. 
We start by fixing some notation we will use in the whole paper.

Let $\mathcal{O}_{2}$ be the local $\mathbb{R}$-algebra of real analytic function germs $\left(\mathbb{R}^{2}, \underline{0}\right) \rightarrow \mathbb{R}$ at the origin $\underline{0}$ of $\mathbb{R}^{2}$. Let $\mathbf{m}_{2}$ be its maximal ideal.

Let $S$ be a real analytic regular surface with structural sheaf $\mathcal{O}_{S}$. Let $\underline{a}$ be a point of $S$. We denote by $\mathcal{O}_{S, \underline{a}}$ the $\mathbb{R}$-algebra of real analytic function germs $(S, \underline{a}) \rightarrow \mathbb{R}$. If $I$ is any (coherent) $\mathcal{O}_{S}$-ideal sheaf, let $I_{\underline{a}}$ be the $\mathcal{O}_{S, \underline{a}}$-ideal induced at $\underline{a}$.

Definition 2.1. A homeomorphism germ $h:\left(\mathbb{R}^{2}, \underline{0}\right) \rightarrow\left(\mathbb{R}^{2}, \underline{0}\right)$ is blow-analytic if there exists a commutative diagram

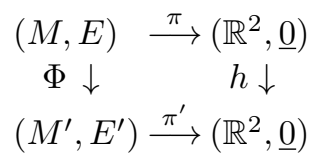

with the following properties

- $\Phi$ is a real analytic isomorphism which induces $h$.

- Both mappings $\pi$ and $\pi^{\prime}$ are finite composition of points blowings-up and $E$ and $E^{\prime}$ are (simple) normal crossing divisors.

Consequently any blow-analytic homeomorphism is sub-analytic. Such homeomorphisms clearly form of sub-group of the homeomorphism germs $\left(\mathbb{R}^{2}, \underline{0}\right) \rightarrow\left(\mathbb{R}^{2}, \underline{0}\right)$.

The blow-analytic equivalence of two function germs of $\mathcal{O}_{2}$ is thus defined as expected:

Definition 2.2. Two real analytic function germs $f, g:\left(\mathbb{R}^{2}, \underline{0}\right) \rightarrow \mathbb{R}$ are blowanalytic equivalent if there exists a blow-analytic homeomorphism $h:\left(\mathbb{R}^{2}, \underline{0}\right) \rightarrow\left(\mathbb{R}^{2}, \underline{0}\right)$ such that $f=g \circ h$.

The blow-analytic equivalence is a right-equivalence, but is indeed an equivalence, which is not obvious at first sight (see Proposition 2 and its Corollary in [13]). A remarkable fact of this equivalence is that it does not admit moduli [13].

An à-priori refinement of the notion of blow-analytic homeomorphism is found in the next

Definition 2.3. A homeomorphism germ $h:\left(\mathbb{R}^{2}, \underline{0}\right) \rightarrow\left(\mathbb{R}^{2}, \underline{0}\right)$ is cascade blowanalytic if there exists a commutative diagram

$$
\begin{aligned}
& \left(M_{k}, E_{k}\right) \stackrel{\pi_{k}}{\longrightarrow}\left(M_{k-1}, E_{k-1}\right) \stackrel{\pi_{k-1}}{\longrightarrow} \cdots \stackrel{\pi_{2}}{\longrightarrow}\left(M_{1}, E_{1}\right) \stackrel{\pi_{1}}{\longrightarrow}\left(\mathbb{R}^{2}, \underline{0}\right) \\
& \Phi \downarrow \quad h_{k-1} \downarrow \quad h_{1} \downarrow \quad h \downarrow \\
& \left(M_{k}^{\prime}, E_{k}^{\prime}\right) \stackrel{\pi_{k}^{\prime}}{\longrightarrow}\left(M_{k-1}^{\prime}, E_{k-1}^{\prime}\right) \stackrel{\pi_{k-1}^{\prime}}{\longrightarrow} \cdots \stackrel{\pi_{2}^{\prime}}{\longrightarrow}\left(M_{1}^{\prime}, E_{1}^{\prime}\right) \stackrel{\pi_{1}^{\prime}}{\longrightarrow}\left(\mathbb{R}^{2}, \underline{0}\right)
\end{aligned}
$$

with the following properties

(i) $\Phi$ is a real analytic isomorphism which induces $h$.

(ii) Each $\pi_{i}$ (resp. $\left.\pi_{j}^{\prime}\right)$ is the blowing-up of a point in $M_{i-1}\left(\right.$ resp. $\left.M_{i-1}^{\prime}\right)$. 
(iii) Each $h_{i}: M_{i} \rightarrow M_{i}^{\prime}$ is a homeomorphism such that $h_{i}\left(E_{i}\right)=E_{i}^{\prime}$.

The condition (iii) implies that the center of the next blowing-up in $M_{i}$ must be mapped by $h_{i}$ onto the center of the next blowing-up in $M_{i}^{\prime}$.

Although a cascade blow-analytic homeomorphism germ seems to be far more rigid than just a blow-analytic homeomorphism germ, it is not so as states the next

Theorem $2.4([\mathbf{1 4}])$. A homeomorphism germ $h:\left(\mathbb{R}^{2}, \underline{0}\right) \rightarrow\left(\mathbb{R}^{2}, \underline{0}\right)$ is blowanalytic if and only if it is cascade blow-analytic.

As we will see in the proof of our main result in Section 5, the property described in Theorem 2.4 is absolutely fundamental, since the combinatorial data controlling the blow-analytic maps and the equivalent functions (once resolved) are depending mostly on the component(s) of the exceptional divisor(s).

A (real analytic) half-branch is the image of the restriction, to the germ at 0 of the non-negative real half-line $\mathbb{R}_{\geq 0}$, of a non constant real analytic map germ $(\mathbb{R}, 0) \rightarrow\left(\mathbb{R}^{2}, \underline{0}\right)$.

Let $\mathcal{C}_{1}$ and $\mathcal{C}_{2}$ be two half-branches. For $r$ positive and small enough, let $\mathbf{S}_{r}$ be the Euclidean sphere centered at $\underline{0}$ of radius $r$. Let $c(r)=\operatorname{dist}\left(\mathcal{C}_{1} \cap \mathbf{S}_{r}, \mathcal{C}_{2} \cap \mathbf{S}_{r}\right)$. The function $c$ is sub-analytic and thus admits a Puiseux expansion of the form

$$
c(r)=r^{\beta} \cdot(\alpha+\phi(r))
$$

where $\beta \in\left(\left[1,+\infty[\cap \mathbb{Q}) \cup\{+\infty\}\right.\right.$; by convention $\beta=+\infty$ if and only if $\mathcal{C}_{1}=\mathcal{C}_{2}$, where $\phi$ is a sub-analytic function germ tending to 0 at 0 and where $\alpha$ is a positive real number. The order of contact between the real analytic half-branches $\mathcal{C}_{1}$ and $\mathcal{C}_{2}$ is the rational number $\beta$.

We are interested in the following properties of blow-analytic homeomorphism germs, which are similar to some of (sub-analytic) bi-Lipschitz homeomorphism germs.

Proposition $2.5([\mathbf{1 4}])$. Let $h:\left(\mathbb{R}^{2}, \underline{0}\right) \rightarrow\left(\mathbb{R}^{2}, \underline{0}\right)$ be a germ of blow-analytic homeomorphism.

(1) The order of contact between real analytic half-branches is preserved by $h$.

(2) There exists constants $0<A<B$ such that for $r$ positive and small enough

$$
A|x| \leq|h(x)| \leq B|x| \text { once }|x| \leq r .
$$

We will show these properties in Section 4 .

\section{Bi-Lipschitz contact equivalence.}

We give here a short account of the (sub-analytic) bi-Lipschitz contact equivalence with a focus on the special case of plane real analytic function germs.

We present an equivalent criterion below, to be used to demonstrate the main result of this note, which, although obvious, was not observed in [4]. 
The contact equivalence between (smooth) mappings, as per se, was first introduced by Mather [16]. The natural extension of Mather's definition to the Lipschitz setting in the function case appeared in $[\mathbf{3}]$, and to the general case in $[\mathbf{1 7}]$

Definition 3.1. Two map-germs $f, g:\left(\mathbb{R}^{n}, \underline{0}\right) \longrightarrow\left(\mathbb{R}^{p}, \underline{0}\right)$ are called $\mathcal{K}$-biLipschitz equivalent (or contact bi-Lipschitz equivalent) if there exist two germs of biLipschitz homeomorphisms $h:\left(\mathbb{R}^{n}, \underline{0}\right) \longrightarrow\left(\mathbb{R}^{n}, \underline{0}\right)$ and $H:\left(\mathbb{R}^{n} \times \mathbb{R}^{p}, \underline{0}\right) \longrightarrow\left(\mathbb{R}^{n} \times \mathbb{R}^{p}, \underline{0}\right)$ such that $H\left(\mathbb{R}^{n} \times\{\underline{0}\}\right)=\mathbb{R}^{n} \times\{\underline{0}\}$ and the following diagram is commutative:

$$
\begin{gathered}
\left(\mathbb{R}^{n}, \underline{0}\right) \stackrel{(i d, f)}{\longrightarrow}\left(\mathbb{R}^{n} \times \mathbb{R}^{p}, \underline{0}\right) \stackrel{\pi_{n}}{\longrightarrow}\left(\mathbb{R}^{n}, \underline{0}\right) \\
h \downarrow \\
H \downarrow \\
\left(\mathbb{R}^{n}, \underline{0}\right) \stackrel{(i d, g)}{\longrightarrow}\left(\mathbb{R}^{n} \times \mathbb{R}^{p}, \underline{0}\right) \stackrel{\pi_{n}}{\longrightarrow}\left(\mathbb{R}^{n}, \underline{0}\right)
\end{gathered}
$$

where $i d: \mathbb{R}^{n} \longrightarrow \mathbb{R}^{n}$ is the identity map and $\pi_{n}: \mathbb{R}^{n} \times \mathbb{R}^{p} \longrightarrow \mathbb{R}^{n}$ is the canonical projection.

The map-germs $f$ and $g$ are called $\mathcal{C}$-bi-Lipschitz equivalent if $h=i d$.

The $\mathcal{R}$-bi-Lipschitz equivalence admits moduli [10], while the $\mathcal{K}$-bi-Lipschitz contact equivalence has none [3]. The following 1-parameter analytic family of real analytic plane function germs

$$
f_{t}:\left(\mathbb{R}^{2}, 0\right) \rightarrow(\mathbb{R}, 0) \text {, defined as }(x, y) \rightarrow f_{t}(x, y)=x^{3}+y^{6}-3 t^{2} x y^{4}, \text { for } t \in \mathbb{R},
$$

has moduli $[\mathbf{1 0}]$, while the sub-family $\left(f_{s}\right)_{0<s<\varepsilon}$ has a single $\mathcal{K}$-bi-Lipschitz class once $\varepsilon$ is small enough, by $[\mathbf{3}]$.

We can think of a $\mathcal{C}$-bi-Lipschitz equivalence as being a family of origin preserving biLipschitz maps of $\mathbb{R}^{p}$ to $\mathbb{R}^{p}$, parameterized by $\mathbb{R}^{n}$ which carry $f(x)$ to $g(x)$ for all $x \in \mathbb{R}^{n}$. If $p=1$, then $\mathbb{R}-\{0\}$ is the union of two contractible sets; each homeomorphism creates a bijection between these components, and this bijection is independent of $x$. This gives an equivalent form for contact equivalence in the bi-Lipschitz category if $p=1$ :

Theorem $3.2([\mathbf{3}])$. Let $f, g:\left(\mathbb{R}^{n}, \underline{0}\right) \rightarrow \mathbb{R}$ be two smooth function germs. The functions $f$ and $g$ are bi-Lipschitz contact equivalent (or $\mathcal{K}$-bi-Lipschitz equivalent) if there exists a bi-Lipschitz homeomorphism germ $h:\left(\mathbb{R}^{n}, \underline{0}\right) \rightarrow\left(\mathbb{R}^{n}, \underline{0}\right)$, there exist non zero, same sign constants $A, B$ and a sign $\sigma \in\{-1,1\}$ such that in a neighborhood of the origin $\underline{0}$ the following inequalities hold true

$$
A \cdot g<\sigma \cdot f \circ h<B \cdot g .
$$

If furthermore $h$ is required to be sub-analytic we will then speak of sub-analytic biLipschitz contact equivalence.

Proof. See Theorem 2.4 of $[\mathbf{3}]$.

The inequality enters into the proof of the theorem as follows. The functions $x \rightarrow$ $f(x) / g(x)$ are bounded by the inequality. Once is checked that the partial derivatives of the function $(x, y) \rightarrow(f(x) / g(x)) \cdot y$, where $y$ is the coordinate on $\mathbb{R}$, are bounded on 
the closed domain (with non-empty interior) $0 \leq|y| \leq|g(x)|$, we deduce that they are Lipschitz on this domain (see $[\mathbf{3}])$. Since $(f / g) \cdot(y \circ g)=f$, the functions $(f / g) \cdot y$ on $0 \leq|y| \leq|g(x)|$ can be used as part of a family of bi-Lipschitz homeomorphisms.

We recall that the bi-Lipschitz contact equivalence of bi-Lipschitz functions has no moduli $[3]$.

Specializing to the case of $f:\left(\mathbb{R}^{2}, \underline{0}\right) \rightarrow \mathbb{R}$, we are going now to recall some material presented in [4]. We will keep it to the minimum needed here.

An arc (at the origin) is the Puiseux series parameterization $\gamma:\left(\mathbb{R}_{\geq 0}, 0\right) \rightarrow\left(\mathbb{R}^{2}, \underline{0}\right)$ of a given real analytic half-branch such that

$$
|\gamma(t)|=t
$$

As an abuse of language we will confuse the notion of arc with its image.

The order of contact between two arcs means the order of contact between the respective half-branch images.

Let $\gamma$ be an arc and let $f \in \mathcal{O}_{2}$ be a real analytic function germ. The function germ $t \rightarrow f \circ \gamma(t)$ is a (converging) Puiseux series written as

$$
f \circ \gamma(t)=t^{\nu}(A+\psi(t))
$$

where $\nu \in] 0,+\infty] \cap \overline{\mathbb{Q}}$, with $\overline{\mathbb{Q}}:=\mathbb{Q} \cup\{+\infty\}$; by convention $\nu=+\infty$ if and only if $f \circ \gamma$ is identically 0 , and otherwise $\psi$ is a sub-analytic function germ tending to 0 at 0 and where $A$ is a non-zero real number. The rational number $\nu_{f}(\gamma):=\nu$ is the normalized order (at the origin) of the function $f$ along the half-branch $\gamma$. The normalized order is the function

$$
\nu_{f}:\{\operatorname{arcs} \text { at } \underline{0}\} \rightarrow \overline{\mathbb{Q}} \text { defined as } \gamma \rightarrow \nu_{f}(\gamma) \text {. }
$$

It has the following properties:

REMARK 3.3. The normalized order of $f$ is always larger than or equal to the multiplicity $m_{f}$ of $f$ at $\underline{0}$. It is also preserved under sub-analytic bi-Lipschitz contact equivalence.

A Hölder triangle, introduced in $[\mathbf{1}]$, is any image of the quadrant $\left(\mathbb{R}_{\geq 0} \times \mathbb{R}_{\geq 0}, \underline{0}\right)$ by a sub-analytic homeomorphism $\left(\mathbb{R}^{2}, \underline{0}\right) \rightarrow\left(\mathbb{R}^{2}, \underline{0}\right)$. The exponent $\beta(T)$ of a given Hölder triangle $T$ is the order of contact at the origin of the boundary curve of $T$ (the images of the axis of the quadrant). By extension and also abuse of language if we speak of a Hölder triangle of exponent $+\infty$, we just mean a half-branch. A second abuse of language is that any neighborhood of the origin is considered as a Hölder triangle with exponent 1 but boundary-less.

REMARK 3.4. Since (sub-analytic) bi-Lipschitz homeomorphisms preserve the order of contact between curves, the image of a Hölder triangle by such an homeomorphism is a Hölder triangle with the same exponent.

Definition 3.5. Let $\gamma$ be an arc, let $f \in \mathcal{O}_{2}$ and let $q=\nu_{f}(\gamma) \geq m_{f}$. 
The width of $f$ along $\gamma$ is the infimum of the exponents taken among all the Hölder triangles $T$ containing the arc $\gamma$ and such that the normalized order $\nu_{f}$ stays constant and equal to $q$ along any arc contained in $T$. This infimum exists and is rational [4]. It is infinite if and only if $f$ vanishes identically along $\gamma$.

We denote this number by $\mu_{f}(\gamma)$.

REMARK 3.6. The width of $f$ along arcs is preserved by the (sub-analytic) biLipschitz contact equivalence.

As can be seen in [4], the notion of width is at the basis of the construction of the complete invariant - minimal pizza - of a sub-analytic bi-Lipschitz contact equivalence class.

The criterion we mention is the following result. We give it in the real analytic category, but it is valid in the category used in [4] for the same reasons as those of the real analytic category.

Proposition 3.7. Two real analytic function germs $f, g:\left(\mathbb{R}^{2}, \underline{0}\right) \rightarrow(\mathbb{R}, 0)$ are (sub-analytically) bi-Lipschitz contact equivalent if and only if there exists a (subanalytic) homeomorphism $h:\left(\mathbb{R}^{2}, \underline{0}\right) \rightarrow\left(\mathbb{R}^{2}, \underline{0}\right)$ such that

(i) for any arc $\gamma$, we get

$$
\nu_{f}(\gamma)=\nu_{g}\left(h_{*} \gamma\right)
$$

where $h_{*} \gamma$ is the arc corresponding to the half-branch $h(\gamma)$.

(ii) for any arc $\gamma$, we get

$$
\mu_{f}(\gamma)=\mu_{g}\left(h_{*} \gamma\right)
$$

Proof. If the functions are already bi-Lipschitz contact equivalent, any equivalence of minimal pizzas is such a homeomorphism (see [4]).

Assume there exists such a sub-analytic homeomorphism. Conditions (i) and (ii) implies that $f$ and $g$ have combinatorially equivalent minimal pizzas [4, Sections 2 and 3], which is sufficient to imply that both functions are sub-analytically bi-Lipschitz contact equivalent.

We do not use that $f, g$ are real analytic, but just that they are sub-analytic and Lipschitz in order to apply [4].

\section{Local normal form at exceptional points.}

This section is devoted to the local form of the blowing down mapping of a finite composition of point blowings-up initiated with the blowing-up of the origin $\underline{0}$ of $\mathbb{R}^{2}$. We can use such semi-local data in order to investigate the asymptotic properties at $\underline{0}$ of the restriction of a function germ $f \in \mathcal{O}_{2}$ to meaningful Hölder triangles (namely in which the function is "monomial").

Let $\pi:(M, E) \rightarrow\left(\mathbb{R}^{2}, \underline{0}\right)$ be a finite composition of points blowings-up. 
Local analytic coordinates $(u, v)$ centered at $\underline{a} \in E$ are called adapted to $E$ at $\underline{a}$ if the following inclusions for germs hold true

$$
\{u=0\} \subset(E, \underline{a}) \subset\{u \cdot v=0\} .
$$

Any local coordinate system at $\underline{a} \notin E$ is adapted to $E$ at $\underline{a}$.

A (coherent) $\mathcal{O}_{M}$-ideal sheaf $\mathcal{I}$ is principal and monomial in $E$ if it is co-supported in $E$ and at any point $\underline{a}$ of $E$, the ideal is locally generated by a monomial in local coordinates adapted to $E$ at $\underline{a}$ :

- if $(u, v)$ are local adapted coordinates at a smooth/regular point a of $E$, that is such that $(E, \underline{a})=\{u=0\}$, we find that $\mathcal{I}_{\underline{a}}$ is locally generated by $u^{p}$ for some non-negative integer $p$;

- if $(u, v)$ are local adapted coordinates at a corner point $\underline{a}$ of $E$, that is such that $(E, \underline{a})=\{u \cdot v=0\}$, we find that $\mathcal{I}_{\underline{a}}$ is locally generated by $u^{p} v^{q}$ for some nonnegative integers $p, q$.

As part of the folklore, we recall the following two facts (both proved by induction on the number of blowings-up):

i) The pull back $I_{\pi}:=\pi^{*}\left(\mathbf{m}_{2}\right)$ of the maximal ideal is principal and monomial in $E$ and its co-support is $E$.

ii) Let $J_{\pi}$ be the ideal generated by the determinant of $\mathrm{d} \pi$. Then it is also principal and monomial and its co-support is also $E$ (see [6, p. 217]).

The local form of $\pi$ at any point of the exceptional divisor is given in the following

Lemma 4.1. Let $\pi:(M, E) \rightarrow\left(\mathbb{R}^{2}, \underline{0}\right)$ be the finite composition of point blowings-up presented above. The mapping $\pi$ has the following properties:

(i) For any non corner point $\underline{a}$ of the normal crossing divisor $E$, there exists $(u, v)$ local adapted coordinates to $E$ at $\underline{a}$ such that $(E, \underline{a})=\{u=0\}$ such that $I_{\pi, \underline{a}}=\left(u^{l}\right)$ and $J_{\pi, \underline{a}}=\left(u^{\mathbf{l}}\right)$ with $\mathbf{l} \geq 2 l-1$. Moreover, (and up to a rotation in the target $\left.\left(\mathbb{R}^{2}, \underline{0}\right)\right)$ we write the mapping $\pi$ at $\underline{a}$ as

$$
(u, v) \rightarrow\left(\alpha u^{l}, u^{l} \phi(u)+\beta u^{m} v\right),
$$

where $l \leq m$ are positive integer numbers and the function $\phi$ is analytic and $\alpha, \beta \in$ $\{-1,+1\}$.

Note that $\mathbf{l}=l+m-1$.

(ii) For any corner point $\underline{a}$ of the normal crossing divisor $E$, there exists $(u, v)$ local adapted coordinates to $E$ at $\underline{a}$ such that $(E, \underline{a})=\{u=0\}$ such that $I_{\pi, \underline{a}}=\left(u^{l} v^{m}\right)$ and $J_{\pi, a}=\left(u^{\mathbf{l}} v^{\mathbf{m}}\right)$ with $\mathbf{l} \geq 2 l-1$ and $\mathbf{m} \geq 2 m-1$. Moreover, (and up to a rotation in the target $\left.\left(\mathbb{R}^{2}, 0\right)\right)$, locally the mapping $\pi$ is written at a as

$$
(u, v) \rightarrow\left(\alpha u^{l} v^{m}, \phi\left(u^{l} v^{m}\right)+\beta u^{n} v^{p}\right)
$$


where $l \leq n$ and $m \leq p$ are positive integer numbers such that the plane vectors $(l, m)$ and $(n, p)$ are not co-linear $((l, m) \wedge(n, p) \neq 0)$, the function $\phi: t \rightarrow t \cdot h\left(t^{1 / e}\right)$ for a real analytic function $h$ and a positive integer $e$, and $\alpha, \beta \in\{-1,+1\}$.

We also see that $\mathbf{l}=l+n-1$ and $\mathbf{m}=m+p-1$.

Proof. By induction on the number of blowings-up. The induction step is true when $(M, E)=\left(\left(\mathbb{R}^{2}, \underline{0}\right), \emptyset\right)$. It is thus sufficient to show that properties (i) and (ii) are preserved under point blowings-up.

Let $\mathbf{p}$ be any point of the exceptional curve $E$. Let $\gamma:\left(M_{1}, E_{1}\right) \rightarrow(M, E)$ be the corresponding blowing-down mapping. Let $D:=\gamma^{-1}(\mathbf{p})$ be the new component of the normal crossing curve $E_{1}$, and we keep denoting $E$ for the strict transform of $E$ by $\gamma$.

First. Assume that the center $\mathbf{p}$ is a regular point of $E$. Suppose we are given coordinates $(u, v)$ centered at $\mathbf{p}$ such that point (i) holds true.

In the chart $(x, y) \rightarrow(x, x y)$, the exceptional curve $D$ has equation $\{x=0\}$. We just write the mapping $\pi \circ \gamma$ as

$$
(x, y) \rightarrow\left(\alpha x^{a}, x^{a} \phi(x)+\beta x^{c} x y\right)=\left(\alpha x^{a}, x^{a} \phi(x)+\beta x^{c+1} y\right),
$$

thus in the form of point (i).

In the chart $(x, y) \rightarrow(x y, y)$, the exceptional curve $D$ has equation $\{y=0\}$. The mapping $\pi$ is written as

$$
(x, y) \rightarrow\left(\alpha x^{a} y^{a}, x^{a} y^{a} \phi(x y)+\beta x^{c} y^{c+1}\right) .
$$

At the point $D \cap E$, we find $x=0$. Thus we obtain a local expression of $\pi$ of type (ii). At a point $\mathbf{p}$ of $D \backslash E$ we have $x=A \neq 0$. We assume for simplicity $A=1$ (in order to avoid discussing on the parity of $a$ and $c$ ). The cases $A$ positive and $A$ negative are dealt with in a very similar way. Let $x:=1+w$ and $y=(1+w)^{-1} z$. This an isomorphism in a neighborhood of the point p. Note that $(D, \mathbf{p})=\{z=0\}$. The mapping $\pi \circ \gamma$ is written as:

$$
(w, z) \rightarrow\left(\alpha z^{a}, z^{a} \phi(z)+\beta(1+w)^{-1} z^{c+1}\right) .
$$

For $|w|<1$, writing $(1+w)^{-1}=1+w^{\prime}$, we see that $\left(w^{\prime}, z\right)$ are also coordinates centered at $\mathbf{p}$. We deduce that the mapping $\pi \circ \gamma$ can be written as:

$$
\left(w^{\prime}, z\right) \rightarrow\left(\alpha z^{a}, z^{a} \theta(z)+\beta z^{c+1} w^{\prime}\right),
$$

where the function $\theta$ is real analytic.

The case of blowing-up a regular point of $E$ has thus been dealt with.

Second. Assume that center $\mathbf{p}$ of the blowing-up is a corner point of $E$. We use the same notations as in the non corner case. There exist adapted coordinates centered at $\mathbf{p}$ such that the mapping $\pi$ in coordinates is:

$$
(u, v) \rightarrow\left(\alpha u^{a} v^{b}, \phi\left(u^{a} v^{b}\right)+\beta u^{c} v^{d}\right) .
$$

In the chart $(x, y) \rightarrow(x, x y)$ the composed mapping $\pi \circ \gamma$ becomes: 


$$
(x, y) \rightarrow\left(\alpha x^{a+b} y^{b}, \phi\left(x^{a+b} y^{b}\right)+\beta x^{d+d} y^{d}\right) .
$$

We observe that the plane vectors $(a+b, b)$ and $(m+n, n)$ are still linearly independent. At the point $D \cap E$, property (ii) is already satisfied as can be seen in Equation (4.8). At a point of $D \backslash E$, we know that $y=A \neq 0$. And we proceed as in the second part of the first case above.

The proof of the Lemma ends just saying that the blowing-up mapping of the origin gives rise to a blowing-down mapping with local expression of the form $(x, y) \rightarrow(x, x y)$ or $(x, y) \rightarrow(x y, y)$, that is of type (i).

REMARK 4.2. 1) The result is clearly true in the complex case as well.

2) The pair of integers $(l, m)$ at a regular point of $E$ is constant along the component of $E$ containing the point, since they are obtained via $I_{\pi}$ and $J_{\pi}$ both principal and monomial in $E$.

At a corner point the numbers $l, m, n, p$ are just from the pairs $(l, n)$ and $(n, p)$ coming from each component through the corner point.

In points (i) and (ii) of Lemma 4.1, the local coordinates $(u, v)$ at $\underline{a}$ are called Hsiang and Pati coordinates at $\underline{a}$ (see $[\mathbf{1 2}],[\mathbf{9}],[\mathbf{2}],[\mathbf{8}]$ ). Following Remark 4.2 we introduce the following.

Definition 4.3. Let $H$ be a component of $E$. The pair of integers $(l, m)$ appearing in the local expression of $\pi$ at (regular) points of $H$ is called the Hsiang and Pati local data of $\pi$ along $H$ (Hsiang and Pati local data for short). The union of all the Hsiang and Pati local data is called Hsiang and Pati data of $\pi$ (Hsiang and Pati data for short).

Hsiang and Pati data allows to describe, up to local quasi-isometry (see also [12], $[\mathbf{9}],[\mathbf{2}])$ the local form at any point point of the exceptional locus $E$ of the pull-back of the Euclidean metric by the "resolution mapping $\pi$ ".

Corollary 4.4. Let $h:\left(\mathbb{R}^{2}, \underline{0}\right) \rightarrow\left(\mathbb{R}^{2}, \underline{0}\right)$ be a cascade blow-analytic homeomorphism

$$
\begin{array}{cc}
(M, E) & \stackrel{\pi^{\prime}}{\longrightarrow}\left(\mathbb{R}^{2}, \underline{0}\right) \\
\Phi \downarrow & h \downarrow \\
\left(M^{\prime}, E^{\prime}\right) \stackrel{\pi}{\longrightarrow}\left(\mathbb{R}^{2}, \underline{0}\right)
\end{array}
$$

We find $\Phi^{*} I_{\pi}=I_{\pi^{\prime}}$ and $\Phi^{*} J_{\pi}=J_{\pi^{\prime}}$.

In particular, the Hsiang and Pati local data of $\pi$ along any component $H$ of $E$ and the Hsiang and Pati local data of $\pi^{\prime}$ along $\Phi(H)$ are equal.

Proof. It is straightforward from the definitions of cascade blow-analytic homeomorphisms and of $I_{\pi}, J_{\pi}, I_{\pi^{\prime}}$ and $J_{\pi^{\prime}}$.

The next result is a consequence of Corollary 4.4 and presents some metric properties of germs of blow-analytic homeomorphisms of the plane. 
Proposition 4.5 (see also $[\mathbf{1 4}])$. Let $h:\left(\mathbb{R}^{2}, \underline{0}\right) \rightarrow\left(\mathbb{R}^{2}, \underline{0}\right)$ be a germ of blowanalytic homeomorphism.

(1) The order of contact between half-branches is preserved by $h$.

(2) There exist constants $0<A<B$ such that for $r$ positive and small enough

$$
A|x| \leq|h(x)| \leq B|x| \text {, once }|x| \leq r .
$$

Proof. The Hsiang and Pati local data of $\pi$ is used to compute explicitly orders of contact.

- Point 1). Let $\gamma_{1}$ and $\gamma_{2}$ be two disjoint arcs at the origin of $\mathbb{R}^{2}$. Let $\Gamma_{i}$ be the strict transform (of the image) of $\gamma_{i}$ by $\pi$.

Claim i). Assume that $\Gamma_{1}$ and $\Gamma_{2}$ intersect a same component $H$ of $E$ at two distinct points, $\underline{a}_{1}$ and $\underline{a}_{2}$. Let $(l, m)$ be the Hsiang and Pati local data of $\pi$ along $H$. The order of contact between $\Gamma_{1}$ and $\Gamma_{2}$ is $\mathrm{m} / \mathrm{l}$.

Proof of Claim i). Let $\underline{b}_{1}, \ldots, \underline{b}_{s}$ be all the corner points of $E$ lying in $H$ indexed in such a way, after having chosen an orientation on $H$, that $\underline{b}_{i}$ is the successor of $\underline{b}_{i-1}$ for $i=2, \ldots, s$. Let $I_{i}$ be the "open interval" $] \underline{b}_{i}, \underline{b}_{i+1}[$.

Assume that $\underline{a}_{1}, \underline{a}_{2} \in I_{i}$ for some $i$. Let $(u, v)$ be local coordinates adapted to $H$. We can parameterize the half-branches $\Gamma_{i}$ as $c_{i}: u \rightarrow\left(u, v_{i}+V_{i}(u)\right)$ where $V_{i}$ is a Puiseux series vanishing at $u=0$ and $v_{1} \neq v_{2}$. We find

$$
\begin{aligned}
\pi\left(c_{i}(u)\right) & =\left(\alpha u^{l}, u^{l} \phi(u)+\beta u^{m}\left(v_{i}+V_{i}(u)\right)\right. \\
& =\left(\alpha t, t g(t)+\beta t^{\frac{m}{l}}\left(v_{i}+W_{i}(t)\right)\right.
\end{aligned}
$$

where $W_{i}$ is a Puiseux series vanishing at $t=0$. Thus the order of contact between $\Gamma_{1}$ and $\Gamma_{2}$ is $l / m$.

Assume now that $\underline{a}_{1}=\underline{b}_{i}$ and $\underline{a}_{2}, \in I_{i-1} \cup I_{i}$. Let $(u, v)$ be local coordinates at $\underline{b}_{i}$ such that $\underline{a}_{2}$ also lies in the domain of the chart. Let $c_{i}: u \rightarrow\left(u, v_{i}+V_{i}(u)\right)$ be a local parameterization of $\Gamma_{1}$ and $\Gamma_{2}$ where $V_{i}$ is a Puiseux series vanishing at $u=0$ and $v_{1}=0 \neq v_{2}$. We see

$$
\begin{aligned}
\pi\left(c_{i}(u)\right) & =\left(\alpha u^{l}\left(v_{i}+V_{i}\right)^{b}, u^{l}\left(v_{i}+V_{i}\right)^{m} g(u, v)+\beta u^{n}\left(v_{i}+V_{i}\right)^{p}\right) \\
& =\left(\alpha t, t g(t)+\beta t^{n / l}\left(v_{i}+V_{i}\right)^{p-(m n / l)}\right) .
\end{aligned}
$$

Thus the order of contact is again $m / l$.

If $\underline{a}_{1} \in\left[\underline{b}_{i}, \underline{b}_{i+1}\right]$ and $\underline{a}_{2} \in\left[\underline{b}_{j}, \underline{b}_{j+1}\right]$ with $|i-j| \geq 2$, the proof is deduced from the cases above using intermediary real analytic arcs $C_{k}$ with $C_{k} \cap H=\underline{a}_{k} \in I_{k}$ for $i<k<j$ (or $j<k<i$ ) to get the order of contact between $\Gamma_{1}$ and $\Gamma_{2}$.

Claim ii). Assume that $\Gamma_{1} \cap E=\left\{\underline{a}_{1}\right\} \in H_{1}$ and $\Gamma_{2} \cap E=\left\{\underline{a}_{2}\right\} \in H_{2}$ such that $\underline{a}_{1} \neq \underline{a}_{2}$ and $H_{1} \cap H_{2}=\{\underline{a}\}$. Let $(l, n)$ and $(m, p)$ be the Hsiang and Pati local data of $\pi$ along $H_{1}$ and respectively along $H_{2}$. The order of contact of $\Gamma_{1}$ and $\Gamma_{2}$ is $\min (n / l, p / m)$. 
Proof of Claim ii). We can assume that $\Gamma_{1}$ and $\Gamma_{2}$ are contained in a chart centered at the corner point $\underline{a}$. Let $C$ be a real analytic half-branch at the origin $\underline{0}$ of $\mathbb{R}^{2}$ such that its strict transform $C^{\prime}$ by $\pi$ intersects with $E$ at $\underline{a}$. The contact between $\Gamma_{1}$ and $\Gamma_{2}$ is the minimum of the orders of contact between $\Gamma_{1}$ and $C^{\prime}$ and between $\Gamma_{2}$ and $C^{\prime}$. In other words, using part i) it is $\min (n / l, p / m)$.

Clatm iii). Suppose $\Gamma_{1} \cap E=\Gamma_{2} \cap E=\{\underline{a}\}$.

- If $\underline{a}$ is not a corner point there are local coordinates $(u, v)$ at a such that $(E, \underline{a})=$ $\{u=0\}$. Let $c_{i}: u \rightarrow\left(u, u^{e_{i}} A_{i}\right)$ be parameterizations of the strict transforms of $\Gamma_{i}$ with $e_{i} \in \mathbb{Q}_{>0}$ and $A_{i}$ is an invertible Puiseux series if not identically 0 , for $i=1,2$. Let $u^{e_{1}} A_{1}-u^{e_{2}} A_{2}=u^{e} A$ for an invertible Puiseux unit $A$. The order of contact between $\Gamma_{1}$ and $\Gamma_{2}$ is $(m+e) / l$.

- If $\underline{a}$ is a corner point, there are local coordinates $(u, v)$ such that $(E, \underline{a})=\{u v=0\}$. Let $c_{i}: u \rightarrow\left(u, u^{e_{i}} A_{i}\right)$ be parameterizations of the strict transforms of $\Gamma_{i}$ with $e_{i} \in \mathbb{Q}_{>0}$ and $A_{i}$ is an invertible Puiseux series if not identically 0 , for $i=1,2$. Let $u^{e_{1}} A_{1}-u^{e_{2}} A_{2}=u^{e} A$ for an invertible Puiseux unit $A$. The order of contact between $\Gamma_{1}$ and $\Gamma_{2}$ is

$$
\frac{n+p \cdot \min \left(e_{1}, e_{2}\right)+e-\min \left(e_{1}, e_{2}\right)}{l+m \cdot \min \left(e_{1}, e_{2}\right)} .
$$

Proof of Claim iii). Assume that $\underline{a}$ is a corner point and that $e_{1} \leq e_{2}$. For simplicity we assume $A_{1}$ and $A_{2}$ are positive. The other cases are dealt with exactly in the same way, just in keeping track of the sign. Then

$$
\begin{aligned}
\pi\left(c_{1}(u)\right) & =\left(\alpha u^{l+m e_{1}} A_{1}^{m}(u), u^{l+m e_{1}} A_{1}^{m}(u) g\left(u^{l+m e_{1}} A_{1}^{m}(u)\right)+\beta u^{n+p e_{1}} A_{1}^{p}(u)\right) \\
& =\left(\alpha t, t h(t)+\beta t^{\left(n+p e_{1}\right) /\left(l+m e_{1}\right)} A_{1}^{(p l-m n) /\left(l+m e_{1}\right)}\left(U_{1}(t)\right)\right) \\
\pi\left(c_{2}(u)\right) & =\left(\alpha u^{l+m e_{2}} A_{2}^{m}(u), u^{l+m e_{2}} A_{2}^{m}(u) g\left(u^{l+m e_{2}} A_{2}^{m}(u)\right)+\beta u^{n+p e_{1}} A_{2}^{p}(u)\right) \\
& =\left(\alpha t, t h(t)+\beta t^{\left(n+p e_{2}\right) /\left(l+m e_{2}\right)} A_{2}^{(p l-m n) /\left(l+m e_{2}\right)}\left(U_{2}(t)\right)\right),
\end{aligned}
$$

where $U_{i}(t)^{l+m e_{i}} A_{i}^{m}\left(U_{i}(t)\right)=t$.

If $e>e_{1}=e_{2}$ then $u^{e_{2}} A_{2}=u^{e_{1}} A_{1}+u^{e} A=u^{e_{1}}\left[A_{1}+u^{e-e_{1}} A\right]$, so that

$\pi\left(c_{1}(t)\right)-\pi\left(c_{2}(t)\left|=t^{\left(n+p e_{1}\right) /\left(l+m e_{1}\right)}\right| A_{1}^{(p l-n m) /\left(l+m e_{1}\right)}\left(U_{1}(t)\right)-A_{2}^{(p l-n m) /\left(l+m e_{1}\right)}\left(U_{2}(t)\right) \mid\right.$.

Since

$$
U_{2}(t)=U_{1}(t)\left[1+t^{\left(e-e_{1}\right) /\left(l+m e_{1}\right)} B(t)\right]
$$

for a Puiseux unit $B_{1}$, we get that the contact between $\Gamma_{1}$ and $\Gamma_{2}$ is $\left(n+p e_{1}+e-e_{1}\right) /\left(l+m e_{1}\right)$. If $e=e_{1}$, a similar but simpler computation gives that the contact is $(n+p e) /(l+m e)$.

Assume $\underline{a}$ is not a corner point. Let $u^{e_{1}} A_{1}-u^{e_{2}} A_{2}=u^{e} A$ with $e \in \mathbb{Q}>0$ and $A$ is an invertible Puiseux series. We find, as above, 


$$
\pi\left(c_{i}(u)\right)=\left(\alpha u^{l}, u^{l} g(u)+\beta u^{m} \cdot u^{e_{i}} A_{i}(u)\right)=\left(\alpha t, \operatorname{tg}(t)+\beta t^{\left(m+e_{i}\right) / l} B_{i}(t)\right)
$$

where $B_{1}$ and $B_{2}$ are invertible Puiseux series. Thus the contact is $(m+e) / l$.

Claim iv). Suppose that $\Gamma_{1} \cap E=\underline{a}_{1} \in H_{1}$ and $\Gamma_{2} \cap E=\underline{a}_{2} \in H_{2}$ with $H_{1} \cap H_{2}=\emptyset$. There is a unique chain $D_{0}, \ldots, D_{s}$ of distinct components of $E$ such that $D_{0}:=H_{1}$, $D_{s}:=H_{2}$ and $D_{i} \cap D_{j}=\emptyset$ if $0 \leq i \leq j-2 \leq s$ and $D_{i} \cap D_{i+1}$ consists in a single corner point. Let $\left(l_{i}, m_{i}\right)$ be the Hsiang and Pati local data of $\pi$ along $D_{i}$. The contact between $\Gamma_{1}$ and $\Gamma_{2}$ is

$$
\min _{i=0, \ldots, s} \frac{m_{i}}{l_{i}}
$$

Proof of Claim iv). This is similar to the case ii).

Following Corollary 4.4 and the expressions of the order of contact given above, the order of contact between two half branches $C_{1}$ and $C_{2}$ is equal to the order of contact of the half-branches $h\left(C_{1}\right)$ and $h\left(C_{2}\right)$.

- Point 2). Let $C$ be a half-branch at the origin $\underline{0}$. Let $\underline{a} \in E$ be the intersection point of the strict transform $C^{\prime}$ of $C$ by $\pi$ with $E$. Let $(u, v)$ be local coordinates at $\underline{a}$ adapted to $E$ so that $\{u=0\} \subset(E, \underline{a}) \subset\{u \cdot v=0\}$.

If $\underline{a}$ is a regular point of $E$, let $(l, m)$ be the Hsiang and Pati local data of $\pi$ at $\underline{a}$, so that $|\pi(u, v)|=|u|^{l} \cdot A(u, v)$ with $A(0,0)>0$.

If it is a corner point, the Hsiang and Pati local data of $\pi$ at $\underline{a}$ consists of two pairs $(l, n)$ and $(m, p)$ and we get $|\pi(u, v)|=|u|^{l}|v|^{m} \cdot A(u, v)$ with $A(0,0)>0$.

Suppose $\underline{a}$ is a corner point. Up to permuting $u$ and $v$, let $c: t \rightarrow\left( \pm t, t^{r} \phi(t)\right)$, with $t \in \mathbb{R}_{\geq 0}$, be a Puiseux parameterization of $C^{\prime}$ such that $\phi$ is an invertible Puiseux series and $r \in \overline{\mathbb{Q}}_{\geq 1}$. Thus

$$
|\pi(c(t))|=t^{l+m r} A(t) \text { for a Puiseux series } A \text { with } A(0)>0,
$$

and

$$
\left|\pi^{\prime}(\Phi(c(t)))\right|=t^{l+m r} B(t) \text { for a Puiseux series } B \text { with } B(0)>0 .
$$

Similar but simpler computations occur at a non corner point.

The above computation says that $L|x| \leq|h(x)| \leq K|x|$ in the image of the considered coordinates chart $(u, v)$, which is a finite union of Hölder triangles and $0<L<K$. By compactness, the exceptional curve $E$ is covered by finitely many such charts. Positive constants $K, L$ can be found such that an inequality of the desired type holds true in a neighborhood of the origin.

REMARK 4.6. Point 1) implies than any blow-analytic image of a Hölder triangle is a Hölder triangle with equal exponent.

\section{Proof of the main result.}

This section is devoted to proof the main result of this note, namely 
THEOREM 5.1. Blow-analytic equivalent real analytic plane function germs are sub-analytically bi-Lipschitz contact equivalent.

Let $f, g \in \mathcal{O}_{2}$ two blow-analytic equivalent function germs. What we exactly show in this section is the following result which, when combined with the results of [4] about abstract pizzas, will yield Theorem 5.1.

Proposition 5.2. Let $h$ be the blow-analytic isomorphism between $f$ and $g$, say $f=g \circ h$. Thus the homeomorphism $h$ maps any pizza of $f$ onto an equivalent pizza of $g$ (although $h$ may never realize any equivalence between any two pizzas).

A non constant function germ $f \in \mathcal{O}_{2}$ is said resolved or monomialized if there exists a finite composition of points blowings-up $\pi:(M, E) \rightarrow\left(\mathbb{R}^{2}, \mathbf{0}\right)$ such that (see $[\mathbf{1 1}],[5],[6])$

- the co-support of the pull-back $I_{f}:=\pi^{*}((f)) \subset I_{\pi}$ of the ideal $(f)$ is a normal crossing divisor $E \cup F$, where $F$ is the strict transform of $f^{-1}(0)$;

- the ideal $I_{f}$ is principal and monomial in $E \cup F$.

Let $f \in \mathcal{O}_{2}$ and let $\pi_{f}:\left(M_{f}, \mathcal{E}_{f}, E_{f}\right) \rightarrow\left(M, f^{-1}(0), \underline{0}\right)$ be a (minimal) resolution of $f$. Since the ideal $I_{f}=\pi_{f}^{*}((f))$ is principal and monomial in the normal crossing divisor $\mathcal{E}_{f}$. Let $\underline{a} \in E_{f}$ and let $(u, v)$ be local coordinates at $\underline{a}$ adapted to $E_{f}$ and $\mathcal{E}_{f}$, that is

$$
\{u=0\} \subset\left(E_{f}, \underline{a}\right) \subset\left(\mathcal{E}_{f}, \underline{a}\right) \subset\{u \cdot v=0\} .
$$

If $\underline{a}$ is a regular point of $\mathcal{E}_{f}$, we get $I_{f, \underline{a}}=\left(u^{r}\right)$ for $r \in \mathbb{N}_{\geq m_{f}}$.

If $\underline{a}$ is a corner point of $\mathcal{E}_{f}$, we have $I_{f, \underline{a}}=\left(u^{r} v^{s}\right)$ for $r, s \in \mathbb{N}_{\geq m_{f}}$.

The number $r$ depends only on the component of $E_{f}$ through the point $\underline{a}$, while the number $s$ depends only on the component of $\mathcal{E}_{f}$ through $\underline{a}$.

DeFinition 5.3. The multiplicity(ies) $r(r, s)$ is (are) called the local resolution data of $f$ at $\underline{a} \in E_{f}$. The (finite) collection of all these integer numbers is called the resolution data of $f$.

Let $f, g \in \mathcal{O}_{2}$ be two blow-analytic equivalent function germs. Let $h$ be the corresponding blow-analytic homeomorphism obtained from the following cascade blowanalytic commutative diagram:

$$
\begin{aligned}
\left(M_{f}, \mathcal{E}_{f}:=\pi_{f}^{-1}(F), E_{f}\right) \stackrel{\pi_{f}}{\longrightarrow}\left(\mathbb{R}^{2}, F:=f^{-1}(0), \underline{0}\right) & \\
& \Phi \downarrow \\
\left(M_{g}, \mathcal{E}_{g}:=\right. & \left.\pi_{g}^{-1}(G), E_{g}\right) \stackrel{\pi_{g}}{\longrightarrow}\left(\mathbb{R}^{2}, G:=g^{-1}(0), \underline{0}\right)
\end{aligned}
$$

where $\mathcal{E}_{f}:=\left\{f \circ \pi_{f}=0\right\}$ and $\mathcal{E}_{g}:=\left\{g \circ \pi_{g}=0\right\}$. Having $f=g \circ h$ is equivalent to have $f \circ \pi_{f}=g \circ \pi_{g} \circ \Phi$, so that $\Phi^{*}\left(\pi_{g}^{*}((g))\right)=\pi_{f}^{*}((f))$.

Any point blowing-up $\tau:(\widetilde{M}, \widetilde{E}) \rightarrow\left(M_{f}, E_{f}\right)$ with center $\mathbf{p}$ in $M_{f}$ leads to a point blowing-up $\tau^{\prime}:\left(\widetilde{M}^{\prime}, \widetilde{E}^{\prime}\right) \rightarrow\left(M_{g}, E_{g}\right)$ with center $\Phi(\mathbf{p})$, so that we can extend $\tau^{*} \Phi$ as an analytic isomorphism $(\widetilde{M}, \widetilde{E}) \rightarrow\left(\widetilde{M}^{\prime}, \widetilde{E}^{\prime}\right)$. This allows to further assume that $\pi_{f}$ 
is a (minimal) resolution of $f$ which (equivalently) implies that $\pi_{g}$ is also a (minimal) resolution of $g$. Under this additional property $\mathcal{E}_{f}$ and $\mathcal{E}_{g}$ are both normal crossing divisor.

The proof of the main result starts with the following

LEMMA 5.4. Let $\gamma$ be a real analytic arc along which $f$ does not vanish identically. Let $\Gamma$ be the strict transform of (the image of) $\gamma$ which intersects with $E_{f}$ at $\underline{a}$. Let $(u, v)$ be local coordinates at a adapted to $E_{f}$ and $\mathcal{E}_{f}$.

The normalized order $\nu_{f}(\gamma)$ of $f$ along $\gamma$ is a fraction whose numerator is affine with coefficients in the local resolution data of $f$ at $\underline{a}$, the denominator is affine with coefficients in the Hsiang and Pati local data of $\pi_{f}$ at $\underline{a}$, and the variable is $p$ as in the parameterization $t \rightarrow\left( \pm t, t^{p} \theta(t)\right)$ of $\Gamma$, with $p \in \mathbb{Q}_{>0}$ and where $\theta$ is an invertible Puiseux series.

Proof. We find below in all possible situations the explicit expression of $\nu_{f}(\gamma)$. Let $\gamma_{f}: t \rightarrow\left( \pm t, t^{p} \theta(u)\right)$ be a Puiseux parameterization of $(\Gamma, \underline{a})$.

- Assume that $\{\underline{a}\}=\Gamma \cap E_{f}$ is a regular point of $E_{f}$ with $\underline{a} \notin \cos \left(\mathcal{E}_{f} \backslash E_{f}\right)$. Since $I_{\pi_{f}, \underline{a}}=\left(u^{l}\right)$ and $I_{f, \underline{a}}=\left(u^{r}\right)$, the normalized order of $f$ along $\gamma$ is

$$
\nu_{f}(\gamma)=\frac{r}{l} .
$$

In this case, we find that

$$
f \circ \gamma_{f}(t)=t^{r} A(t)
$$

for an invertible Puiseux series $A$. Since $\left|\pi_{f} \circ \gamma_{f}(t)\right|=|t|^{l} B(t)$ for a positive Puiseux series $B$, we get the results.

- Assume that $\underline{\text { a }}$ is a corner point of $\mathcal{E}_{f}$ but a regular point of $E_{f}$. Thus $I_{\pi_{f}, \underline{a}}=\left(u^{l}\right)$ and $I_{f, \underline{a}}=\left(u^{r} v^{s}\right)$. The normalized order of $f$ along $\gamma$ is

$$
\nu_{f}(\gamma)=\frac{r+s p}{l}
$$

We find

$$
f \circ \gamma_{f}(t)=t^{r+p s} A(t) \text { and }\left|\pi_{f} \circ \gamma_{f}(u)\right|=|t|^{l} B(t)
$$

for invertible Puiseux series $A, B$. So we deduce the normalized order.

- Assume that $\{\underline{a}\}=\Gamma \cap E_{f}$ is a corner point of $E_{f}$, so that $I_{\pi_{f}, \underline{a}}=\left(u^{l} v^{m}\right)$ and $I_{f, \underline{a}}=\left(u^{r} v^{s}\right)$. The normalized order of $f$ along $\gamma$ is

$$
\nu_{f}(\gamma)=\frac{r+s p}{l+m p}=\frac{r q+s}{l q+m} .
$$

This last situation yields

$$
f \circ \gamma_{f}(t)=t^{r+p s} A(t) \text { and }\left|\pi_{f} \circ \gamma_{f}(t)\right|=|t|^{l+p m} B(t)
$$


for invertible Puiseux series $A, B$. The case of a parameterization of the form $\tau \rightarrow\left(\tau^{q} \mu(\tau), \pm \tau\right)$ works similarly.

A key to our proof consequence of Lemma 5.4 is the following

Proposition 5.5. Let $\gamma$ be an arc and let $h_{*} \gamma$ be the arc corresponding to the image $h \circ \gamma$. We find

$$
\nu_{f}(\gamma)=\nu_{g}\left(h_{*} \gamma\right)
$$

Proof. The function $f$ vanishes identically along $\gamma$ if and only if $g$ vanishes identically along $h_{*} \gamma$.

Assume that $\gamma$ is not contained in $f^{-1}(0)$, thus $h_{*} \gamma$ is not contained in $g^{-1}(0)$ either.

Following Section 4, such as Corollary 4.4, all the integers linked to $\pi_{f}, f$ and $\Gamma$ used to compute the normalized order of $f$ along the arc $\gamma$ in Lemma 5.4 are inherited by $\pi_{g}, g$ and $\Phi(\Gamma)$ via the analytic isomorphism $\Phi$.

Combining the fact that, any blow-analytic image of a Hölder triangle is a Hölder triangle of equal exponent, with Proposition 5.5 the proof of our main result is, in principle, already "done". We are now ready to go into the

Proof of Proposition 5.2. From Lemma 5.4 and Proposition 5.5 we deduce that the equivalent criterion of sub-analytic bi-Lipschitz contact equivalence presented as Proposition 3.7 is satisfied, concluding the proof.

\section{Appendix: a sketch of the existence of Pizzas.}

We present here a sketch of the existence of pizza (in the case of a plane real analytic function germ).

The paper [4] uses a general Preparation Theorem for the functions considered there (more general than continuous sub-analytic plane function germs), which, roughly speaking, states that the function behaves like a monomial in some Hölder-like triangle, so that we can find finitely many of them to cover a neighborhood of the considered point. In the case of plane real analytic function germs, and in relation with blowanalytic equivalence we are going to use here the resolution of singularities of the given function in order to provide explicit, in the combinatorial data (function and resolution mapping), expressions for the width.

Let $f \in \mathcal{O}_{2}$ be given and let $\pi:\left(M_{f}, \mathcal{E}_{f}, E_{f}\right) \rightarrow\left(E, f^{-1}(0), \underline{0}\right)$ be the (minimal) resolution of $f$.

Let $\underline{a}$ be a point of $E_{f}$ and let $(u, v)$ local coordinates at $\underline{a}$ adapted to $E_{f}$ and $\mathcal{E}_{f}$ so that

$$
\{u=0\} \subset\left(E_{f}, \underline{a}\right) \subset\left(\mathcal{E}_{f}, \underline{a}\right) \subset\{u \cdot v=0\} .
$$

We also know that

$$
I_{\pi, \underline{a}}=\left(u^{l} v^{m}\right) \text { and } J_{\pi, \underline{a}}=\left(u^{\mathbf{l}} v^{\mathbf{m}}\right) \text { and } I_{f, \underline{a}}=\left(u^{r} v^{s}\right) .
$$


With certainty we know that $\mathbf{l} \geq 2 l-1 \geq 1$, and $\mathbf{m} \geq 2 m-1 \geq 1$ and $r \geq m_{f} \cdot l$. We can assume that we are working in a (semi-analytic) box $\left.B_{\underline{a}}:=\right]-u_{\underline{a}}, u_{\underline{a}}[\times]-v_{\underline{a}}, v_{\underline{a}}[$ for positive real number $u_{\underline{a}}$ and $v_{\underline{a}}$.

The image $\pi\left(\operatorname{clos}\left(\bar{B}_{\underline{a}}\right)\right)$ is a finite union of (one, two, or four) Hölder triangle(s) (with equal exponent) which can be calculated by the Hsiang and Pati local data of $\pi$ at $\underline{a}$.

We can check that $f$ is monotonic on each such Hölder triangle, and with computations similar (but longer) to those done in the proofs of Proposition 4.5 and of Lemma 5.4 we can explicitly find the expression of the (non directed) width $\omega_{f}^{T}$ in terms of the exponents $l, m, \mathbf{l}, \mathbf{m}, r, s$.

Since $E_{f}$ is compact we can cover it with finitely many closure of (semi-analytic) boxes of the type $B_{\underline{a}}$ above in such a way the intersection of the closures of two such boxes has always empty interior. This partition provides the triangulation of a neighborhood of the origin by finitely many Hölder triangles (the images by the resolution mapping $\pi$ of the closures of all the boxes) thus and pizza adding the (directed) width and the signs of the function within the interior of these Hölder triangles.

Let us make a final comment:

REMARK 6.1. Either way (Preparation Theorem or minimal resolution) picked to cook-up a pizza of a plane real analytic function germ, the combinatorial data to handle to describe the width functions may be huge. Thus, when the function has a simpler combinatorial data (such as a non-degenerate Newton diagram) it should be possible to describe, by means of this simpler combinatorial data, pizzas attached to $f$.

\section{References}

[1] L. Birbrair, Local bi-Lipschitz classification of 2-dimensional semi-algebraic sets, Houston J. Math., 25 (1999), 453-472.

[2] A. Belotto, E. Bierstone, V. Grandjean and P. Milman, Resolution of the singularities of the cotangent sheaf of a singular variety, Adv. Math., 307 (2017), 780-832.

[ 3 ] L. Birbrair, J.-C. Costa, A. Fernandes and M. A. Ruas, $\mathcal{K}$-bi-Lipschitz equivalence of real functiongerms, Proc. Amer. Math. Soc., 135 (2007), 1089-1095.

[4] L. Birbrair, A. Fernandes, A. Gabrielov and V. Grandjean, Lipschitz contact equivalence of function germs in $\mathbb{R}^{2}$, Ann. Sc. Norm. Super. Pisa Cl. Sci. (5), XVII (2017), 81-92.

[5] E. Bierstone and P. Milman, Semi-analytic and sub-analytic sets, Inst. Hautes Études Sci. Publ. Math., 67 (1988), 5-42.

[6] E. Bierstone and P. Milman, Canonical desingularization in characteristic zero by blowing up the maximum strata of a local invariant, Invent. Math., 128 (1997), 207-302.

[ 7 ] T. Fukuda, Types topologiques des polynômes, IHÉS. Publ. Math., 46 (1976), 87-106.

[8] V. Grandjean, Monomialization of singular metrics on real surfaces, preprint 2015, available at http://arxiv.org/abs/1505.05167

[ 9 ] D. Grieser, Hsiang and Pati coordinates for real analytic isolated surface singularities, Personal notes (2000) 4 pages.

[10] J.-P. Henry and A. Parusiński, Existence of moduli for bi-Lipschitz equivalence of analytic functions, Compositio Math., 136 (2003), 217-235.

[11] H. Hironaka, Resolution of singularities of an algebraic variety over a field of characteristic zero, Ann. of Math., 79 (1964), 109-326.

[12] W. C. Hsiang and V. Pati, $L^{2}$-cohomology of normal algebraic surfaces, Invent. Math., 81 (1985), 395-412.

[13] T. Z. Kuo, On classification of real singularities, Invent. Math., 82 (1985), 257-262. 
[14] S. Koike and A. Parusiński, Blow-analytic equivalence of two variables real analytic function germs, J. Algebraic Geometry, 19 (2010), 439-472.

[15] S. Koike and A. Parusiński, Equivalence relations for two variable real analytic function germs, J. Math. Soc. Japan, 65 (2013), 237-276.

[16] J. Mather, Stability of $C^{\infty}$ mappings III: Finitely determined map-germs, Inst. Hautes Études Sci. Publ. Math., 35 (1968), 279-308.

[17] M. A. Ruas and G. Valette, $C^{0}$ and bi-Lipschitz K-equivalence of mappings, Math. Z., 269 (2011), 293-308.

\section{Lev BIRBRAIR}

Departamento de Matemática Universidade Federal do Ceará Av. Humberto Monte s/n Campus do Pici - Bloco 914 60455-760 Fortaleza-CE, Brazil E-mail: birb@ufc.br

\section{Vincent GRANDJEAN}

Departamento de Matemática Universidade Federal do Ceará Av. Humberto Monte s/n Campus do Pici - Bloco 914 60455-760 Fortaleza-CE, Brazil E-mail: vgrandjean@mat.ufc.br

\author{
Alexandre Fernandes \\ Departamento de Matemática \\ Universidade Federal do Ceará \\ Av. Humberto Monte \\ s/n Campus do Pici - Bloco 914 \\ 60455-760 Fortaleza-CE, Brazil \\ E-mail: alex@mat.ufc.br
}

\section{Terence Gaffney}

Mathematics department Northeastern University 360 Huntington Ave. 02115 Boston-MA, USA

E-mail: t.gaffney@neu.edu 